\title{
A NOTE ON FUNCTIONS OF EXPONENTIAL TYPE ${ }^{1}$
}

R. P. BOAS, JR.

An entire function $f(z)$ is said to be of exponential type at most $T$ if

$$
\limsup _{n \rightarrow \infty}\left|f^{(n)}(z)\right|^{1 / n} \leqq T
$$

for some $z$ (and hence for every $z$, uniformly for $z$ in any bounded set). An equivalent condition ${ }^{2}$ is that for each positive $\epsilon$

$$
|f(z)|<e^{(T+\epsilon)|z|}
$$

for all sufficiently large $|z|$. The following three theorems were proved respectively by D. V. Widder [4], I. J. Schoenberg [2], and H. Poritsky [1] and J. M. Whittaker [3].

THEOREM 1. (Widder.) If a real function $f(z)$, of class $C^{\infty}$ in $0 \leqq x \leqq 1$, satisfies the condition

$$
(-1)^{n} f^{(2 n)}(x) \geqq 0, \quad 0 \leqq x \leqq 1 ; n=0,1,2, \cdots,
$$

then $f(x)$ coincides on $(0,1)$ with an entire function of exponential type at most $\pi$.

THEOREM 2. (Schoenberg.) If $f(z)$ is an entire function of exponential type at most $T$, and if

$$
f^{(2 n)}(0)=f^{(2 n)}(1)=0, \quad n=0,1,2, \cdots,
$$

then $f(z)$ is a sine polynomial of order at most $T / \pi$ :

$$
f(z)=\sum_{k=0}^{N} a_{k} \sin k \pi z, \quad N \leqq T / \pi .
$$

Let $\Lambda_{n}(z)$ be the polynomial of degree $2 n+1$ determined by the relations

$$
\begin{aligned}
& \Lambda_{0}(z)=z ; \quad \Lambda_{n}(0)=\Lambda_{n}(1)=0, \quad n \geqq 1 ; \\
& \Lambda_{n}^{\prime \prime}(z)=\Lambda_{n-1}(z), \quad n \geqq 1 .
\end{aligned}
$$

THEOREM 3. (Poritsky-Whittaker.) If $f(z)$ is an entire function of exponential type at most $T, T<\pi$, then $f(z)$ can be represented in the form

1 Presented to the Society, February 22, 1941.

${ }^{2}$ G. Valiron, Lectures on the General Theory of Integral Functions, Toulouse, 1923, p. 41. 


$$
f(z)=\sum_{n=0}^{\infty} f^{(2 n)}(1) \Lambda_{n}(z)-\sum_{n=0}^{\infty} f^{(2 n)}(0) \Lambda_{n}(z-1),
$$

where the series converge uniformly in any bounded region.

An expansion of the form (4) is called a Lidstone series.

The chief purpose of this note is to give a short proof of Theorem 1 which I obtained some time after Professor Widder communicated the theorem to me. ${ }^{3}$ I also give simple proofs of Theorems 2 and 3, suggested by the proof of Theorem 1 .

LEMMA 1. If $k$ and $n$ are positive integers, and $f(x)$ is of class $C^{2 n}$ in $0 \leqq x \leqq 1$, then

$$
\begin{aligned}
\int_{0}^{1} f(x) \sin k \pi x d x= & \sum_{m=0}^{n-1} \frac{(-1)^{m}}{(k \pi)^{2 m+1}}\left\{f^{(2 m)}(0)-(-1)^{k} f^{(2 m)}(1)\right\} \\
& +\frac{(-1)^{n}}{(k \pi)^{2 n}} \int_{0}^{1} f^{(2 n)}(x) \sin k \pi x d x
\end{aligned}
$$

This is proved by integrating the left-hand side repeatedly by parts.

LEMMA 2. If $g(x)$ is non-negative and concave in $a \leqq x \leqq b$, and

then

$$
\int_{a}^{b} g(x) d x \leqq A
$$

$$
g(x) \leqq \frac{2 A}{b-a}, \quad a \leqq x \leqq b .
$$

Let $g(x)$ take its maximum, $G$, at $x=x_{0}$. Since $g(x)$ is concave, the graph of $g(x)$ is above the broken line connecting the points $(a, 0)$ and $(b, 0)$ to $\left(x_{0}, G\right)$. The area under the broken line is $\frac{1}{2} G(b-a)$. Hence $\frac{1}{2} G(b-a) \leqq A$, and (6) follows.

Proof of Theorem 1. Take $k=1$ in Lemma 1 . By hypothesis the terms of the sum on the right of (5) are all non-negative. Hence, for any positive integer $n$,

$$
\begin{array}{cr}
B=\int_{0}^{1} f(x) \sin \pi x d x \geqq \frac{1}{\pi^{2 n}} \int_{0}^{1}(-1)^{n} f^{(2 n)}(x) \sin \pi x d x \geqq 0, \\
\int_{\delta}^{1-\delta}(-1)^{n} f^{(2 n)}(x) \sin \pi x d x \leqq B \pi^{2 n}, & 0<\delta<\frac{1}{2},
\end{array}
$$

${ }^{3}$ (Added in proof.) Essentially the same proof was found independently by Professor Schoenberg. 


$$
\int_{\delta}^{1-\delta}(-1)^{n} f^{(2 n)}(x) d x \leqq \frac{B \pi^{2 n}}{\sin \pi \delta} .
$$

Since $(-1)^{n} f^{(2 n+2)}(x) \leqq 0,(-1)^{n} f^{(2 n)}(x)$ is non-negative and concave in $(\delta, 1-\delta)$. By Lemma 2 ,

$$
0 \leqq(-1)^{n} f^{(2 n)}(x) \leqq \frac{2 B \pi^{2 n}}{(1-2 \delta) \sin \pi \delta}, \quad \delta \leqq x \leqq 1-\delta .
$$

Let $h=\frac{1}{2}-\delta$. For any $x$ in $(\delta, 1-\delta)$, Taylor's theorem with remainder of order 2 , applied to $f^{(2 n)}(x)$, leads to the relation

$$
\begin{array}{r}
f^{(2 n+1)}(x)= \pm \frac{1}{h}\left[f^{(2 n)}(x \pm h)-f^{(2 n)}(x)\right] \mp \frac{1}{2} h f^{(2 n+2)}(x \pm \theta h), \\
0<\theta<1,
\end{array}
$$

where the upper signs or the lower signs are taken according as $x+h$ or $x-h$ is in $(\delta, 1-\delta)$. Using (7), written both for $n$ and $n+1$, we obtain

$$
\left|f^{(2 n+1)}(x)\right| \leqq\left(\frac{2}{h}+\frac{\pi^{2} h}{2}\right) \frac{2 B \pi^{2 n}}{(1-2 \delta) \sin \pi \delta}=C(\delta) B \pi^{2 n+1},
$$

where $C(\delta)$ depends only on $\delta$. Thus

$$
\limsup _{n \rightarrow \infty}\left|f^{(n)}(x)\right|^{1 / n} \leqq \pi,
$$

uniformly in any interval $\delta<x<1-\delta$, where $0<\delta<\frac{1}{2}$. The relation (8) implies first that the Taylor series of $f(x)$ about any point in $(0,1)$ converges to an entire function coinciding with $f(x)$ in $(0,1) ;(8)$ then shows that this function is of exponential type at most $\pi$.

Proof of Theorem 2. Since $f(x)$ is of class $C^{\infty}$, it is represented in $(0,1)$ by its Fourier sine series:

$$
f(x)=\sum_{k=0}^{\infty} a_{k} \sin k \pi x, \quad 0<x<1,
$$

where

$$
a_{k}=2 \int_{0}^{1} f(t) \sin k \pi t d t .
$$

Since (3) is true, Lemma 1 shows that

$$
a_{k}=\frac{2(-1)^{n}}{(k \pi)^{2 n}} \int_{0}^{1} f^{(2 n)}(t) \sin k \pi t d t
$$

for every positive integer $n$. Hence 


$$
\left|a_{k}\right| \leqq \frac{2}{(k \pi)^{2 n}} \max _{0 \leqq x \leqq 1}\left|f^{(2 n)}(x)\right| .
$$

If $k \pi>T$, for large $n$ we have

$$
\max _{0 \leqq x \leqq 1}\left|f^{(2 n)}(x)\right| \leqq S^{2 n}, \quad T<S<k \pi,
$$

since $f(x)$ is of exponential type at most $T$. From (10) it then follows that $a_{k}=0$ if $k \pi>T$. Thus all terms of (9) with $k>T / \pi$ vanish, and Theorem 2 is proved.

Proof of THEOREM 3. The function $f(x)$ is represented in $(0,1)$ by the Fourier series (9), and $\frac{1}{2} a_{k}$ is just the integral on the left of (5). Hence, for every positive integer $n$,

$$
\begin{aligned}
f(x)= & \sum_{m=0}^{n-1} f^{(2 m)}(1) \sum_{k=1}^{\infty} \frac{2(-1)^{k+m+1} \sin k \pi x}{(k \pi)^{2 m+1}} \\
& -\sum_{m=0}^{n-1} f^{(2 m)}(0) \sum_{k=1}^{\infty} \frac{2(-1)^{m+1} \sin k \pi x}{(k \pi)^{2 m+1}}+\frac{1}{2} R_{n}(x),
\end{aligned}
$$

where

$$
R_{n}(x)=(-1)^{n} \int_{0}^{1} f^{(2 n)}(t) \sum_{k=1}^{\infty} \frac{\sin k \pi x \sin k \pi t}{(k \pi)^{2 n}} d t .
$$

The infinite series appearing in (11) are the Fourier sine series of $\Lambda_{m}(x)$ and $\Lambda_{m}(1-x)$, as given by Whittaker; ${ }^{4}$ they can easily be checked by successive integrations of the well known Fourier sine series of $\Lambda_{0}(x)=x$. Relation (11) becomes

$$
f(x)=\sum_{m=0}^{n-1} f^{(2 m)}(1) \Lambda_{m}(x)-\sum_{m=0}^{n-1} f^{(2 m)}(0) \Lambda_{m}(x-1)+R_{n}(x) .
$$

We have thus obtained a "Lidstone series with remainder," with an expression for the remainder as a real in tegral equivalent to that given by Widder [4].

We have

$$
\left|R_{n}(x)\right| \leqq \frac{1}{\pi^{2 n}} \sum_{k=1}^{\infty} \frac{1}{k^{2 n}} \int_{0}^{1}\left|f^{(2 n)}(t)\right| d t,
$$

and this approaches zero as $n \rightarrow \infty$, uniformly in $(0,1)$, if $f(z)$ satisfies (1) with $T<\pi$. Hence under the hypothesis of Theorem 3 the series in (4) converges to $f(x)$ uniformly in $(0,1)$. Now Whittaker has

$[3$, p. 454]. 
shown ${ }^{5}$ that if a Lidstone series converges for some non-integral value of $z$, it converges for all $z$, uniformly in any bounded region, and so represents an entire function, which in our case must be $f(z)$. This completes the proof of Theorem 3.

By applying, instead of Lemma 1, the formula obtained by integrating

$$
\int_{0}^{1} f(x) \cos \frac{1}{2} k \pi x d x
$$

by parts, we can prove Schoenberg's theorem [2], analogous to Theorem 2 , that a function $f(z)$ of exponential type is a cosine polynomial if $f^{(2 n)}(1)=f^{(2 n+1)}(0)=0(n=0,1,2, \cdots)$; and we can obtain Whittaker's result [3] corresponding to Theorem 3, concerning the expansion of $f(z)$ in a series with coefficients $f^{(2 n)}(1), f^{(2 n+1)}(0)$. The analogue of Theorem 1 is

THEOREM 4. If $f(x)$ is of class $C^{\infty}$ in $0 \leqq x \leqq 1$, and

$$
\begin{aligned}
f^{(4 n)}(x) \geqq 0, \quad(-1)^{n} f^{(2 n)}(1) \geqq 0, \quad(-1)^{n} f^{(2 n+1)}(0) & \leqq 0, \\
n & =0,1,2, \cdots,
\end{aligned}
$$

then $f(x)$ coincides over $(0,1)$ with an entire function of exponential type at most $\pi / 2$.

\section{REFERENCES}

1. H. Poritsky, On certain polynomial and other approximations to analytic functions, Transactions of this Society, vol. 34 (1932), pp. 274-331; p. 287.

2. I. J. Schoenberg, On certain two-point expansions of integral functions of exponential type, this Bulletin, vol. 42 (1936), pp. 284-288.

3. J. M. Whittaker, On Lidstone's series and two-point expansions of analytic functions, Proceedings of the London Mathematical Society, (2), vol. 36 (1933-1934), pp. 451-469.

4. D. V. Widder, Functions whose even derivatives have a prescribed sign, Proceedings of the National Academy of Sciences, vol. 26 (1940), pp. 657-659.

Duke University

5 [3, p. 455]. 